Eur Phys J C (2003)

Digital Object Identifier (DOI) 10.1140/epjcd/s2003-03-803-y

\title{
Searches for Neutral Higgs Boson and Interpretations in the MSSM at LEP
}

\author{
Philip Bechtle ${ }^{\mathrm{a}}$ \\ DESY, Notkestr. 85, 22607 Hamburg, Germany; E-mail: philip.becht le@desy · de
}

Received: 1 Oct 2003 / Accepted: 20 Nov 2003 /

Published Online: 26 Nov 2003 - (c) Springer-Verlag / Società Italiana di Fisica 2003

\begin{abstract}
This paper discusses recent publications of the LEP collaborations DELPHI, L3 and OPAL on searches for Higgs bosons motivated by MSSM scenarios as well as their interpretation in the MSSM. With the final publication of the LEP collaborations available or awaited, more and more interpretations in different MSSM models, including both $\mathrm{CP}$ conserving and $\mathrm{CP}$ violating, become available. Also specialized analyses close open areas in the parameter space. In the same time, better theoretical calculations with an increased maximal mass of the $h$ boson were presented. Both the new scenarios as well as the new theoretical limit on $m_{\mathrm{h}}$ has consequences for the limits from LEP. The searches, the models in which they are interpreted and the implications of the LEP results for future SUSY searches, especially on the $\tan \beta$ limit, are presented here.
\end{abstract}

PACS. 12.60.Fr Extensions of electroweak Higgs sector - 12.60.Jv Supersymmetric models - 13.66.Fg Gauge and Higgs boson production in $\mathrm{e}^{+} \mathrm{e}^{-}$interactions - 14.80.Cp Non-standard-model Higgs bosons

\section{Introduction}

In the Standard Model (SM) it is generally assumed that the Higgs mechanism is resposible for the breaking of electroweak symmetry and for the generation of elementary particle masses. The Minimal Supersymmetric Standard Model (MSSM) is the SUSY extension of the SM with minimal new particle content. It introduces two complex Higgs field doublets. The MSSM predicts five Higgs bosons: three neutral and two charged ones. At least one of the neutral Higgs bosons is predicted to have its mass close to the electroweak energy scale, providing a high motivation to the searches at current and future colliders.

In the MSSM the Higgs potential is assumed to be invariant under CP transformation at tree level. However, it is possible to break CP symmetry in the Higgs sector by radiative corrections, especially by contributions from complex trilinear couplings $A_{\mathrm{t}, \mathrm{b}}$ of third generation scalar-quarks [1].

Since the input parameter space is generally too large to be scanned completely, so called benchmark scenarios (cf. Tab.1) have been proposed [2 34], each emphasising a certain phenomenological situation. The parameters $\tan \beta=v_{2} / v_{1}$ and $m_{\mathrm{A}}$ governing the Higgs sector on tree-level are scanned, while all parameters on loop level are kept fixed for one scenario. CP conserving (CPC) and $\mathrm{CP}$ violating (CPV) scenarios exist.

Depending on the parameters of the MSSM, Higgs Bosons can be produced in Higgstrahlung $\mathrm{e}^{+} \mathrm{e}^{-} \rightarrow \mathrm{hZ}$, as in the SM, or in pair production $\mathrm{e}^{+} \mathrm{e}^{-} \rightarrow \mathrm{hA}$. Flavour independent Higgs decays, Higgs decays into invisible particles or decays of the type $\mathrm{h} \rightarrow \mathrm{AA}$ additionally present new topologies.

\footnotetext{
a Speaker; on behalf of the LEP collaborations
}

Table 1. The MSSM scenarios used by the LEP collaborations, proposed in [2], [3] and [4]

\begin{tabular}{|c|c|}
\hline \multicolumn{2}{|r|}{$\mathrm{CP}$ conserving } \\
\hline No Mixing & No mixing in the stop-sbottom sector \\
\hline $\mathrm{m}_{h} \max$ & Maximum $m_{\mathrm{h}}$ for given $\tan \beta, m_{\mathrm{A}}$ \\
\hline Large $\mu$ & $\begin{array}{l}\text { Always kinematically accessible, } \\
\text { but } \mathrm{h} \rightarrow \mathrm{b} \overline{\mathrm{b}} \text { suppressed }\end{array}$ \\
\hline $\mathrm{m}_{h} \max ^{+}$ & like $\mathrm{m}_{h}$ max, but favoured by $(g-2)_{\mu}$ \\
\hline constr. $\mathrm{m}_{h} \max$ & like $\mathrm{m}_{h}$ max, but favoured by $(\mathrm{b} \rightarrow \mathrm{s} \gamma)$ \\
\hline gluophobic & $\begin{array}{l}\text { hgg coupling suppressed, } \\
\text { reduced LHC production cross section }\end{array}$ \\
\hline small $\alpha_{\mathrm{eff}}$ & $\begin{array}{l}\mathrm{h} \rightarrow \mathrm{b} \overline{\mathrm{b}} \text { suppressed by cancellation of } \\
\tilde{\mathrm{b}}-\tilde{\mathrm{g}} \text { loops }\end{array}$ \\
\hline \multicolumn{2}{|r|}{$\mathrm{CP}$ violating } \\
\hline CPX & $\begin{array}{l}\text { Mixing of CP- and mass-eigensates } \\
\text { several derivates under study }\end{array}$ \\
\hline
\end{tabular}

Since the end of LEP running, the LEP collaborations have developed new searches closing some of these unexcluded areas in the parameter space, thereby giving important new information about the tasks left over for future experiments. This publication will focus on new searches dedicated to formerly uncovered final states described in Section 2 on the consequences of new theoretical developments in Section 3 and on the interpetation of the MSSM Higgs searches in the benchmark scenarios in Section 4 


\section{Searches for Higgs bosons in the MSSM}

The search for Higgs bosons in the MSSM uses a large variety of channels.The SM production channels Higgsstrahlung $\mathrm{e}^{+} \mathrm{e}^{-} \rightarrow \mathrm{hZ}$ and Boson fusion are reintrerpreted in the MSSM. Additionally, dedicated searches for Higgsstrahlungschannels with Higgs decays in the MSSM exist. Also, pair production $\mathrm{e}^{+} \mathrm{e}^{-} \rightarrow \mathrm{hA}$ and Yukawa production $\mathrm{e}^{+} \mathrm{e}^{-} \rightarrow \mathrm{bb} h / \mathrm{A}$ channels are used. New searches comprise the search for $\mathrm{e}^{+} \mathrm{e}^{-} \rightarrow \mathrm{hZ}$ with $\mathrm{h} \rightarrow \mathrm{AA}$, with $m_{\mathrm{A}}<10 \mathrm{GeV}$ below the b $\overline{\mathrm{b}}$ production threshold [5] by OPAL. The same final state with heavier $m_{\mathrm{A}}$ has been sought-after for the interpretation in CPV models [6].

The search from DELPHI [7] for invisibly decaying Higgs bosons has been interpreted in a modified $m_{\mathrm{h}}$-max scenario with $M_{2}=\mu=150 \mathrm{GeV}$. It shows that the benchmark scenarios from Tab. 1] do not cover the full range of possible MSSM topologies in the Higgs sector, since the search for invisibly decaying Higgs bosons is needed to cover areas unexcluded by the standard searches.

\section{New benchmark scenarios and Higgs mass calculations in the MSSM}

The calculations of the observables of the Higgs sector, the masses, branching ratios and cross-sections, depending on the choice of SUSY parameters have been performed using two different calculation tools. FEYNHIGGS [8,9] is based on the two-loop diagrammatic approach of [10], and SUBH$\mathrm{POLE} / \mathrm{CPH}[4]$ is based on the one-loop renormalization-group improved calculation of [11,12].

The first three CPC benchmark scenarios of Tab. 1 1have traditionally been considered in the past and have now been extended to scenarios 4 and 5, motivated by limits on the branching ratio of the inclusive decay of a $\mathrm{B}$ meson into strange particle states and a photon $\mathrm{B} \rightarrow \mathrm{X}_{\mathrm{s}} \gamma$ and muon anomalous magnetic moment $(g-2)_{\mu}$ measurements. The last two CPC benchmark scans are aiming to set the stage for future Higgs searches at the LHC. There, some of the dominant search channels would be suppressed, resulting in a reduced search sensitivity. The CPV scenario CPX maximises the mixing of CP- and masseigenstates and has been tested wit hseveral different parameter settings [6].

With respect to the calculations used for the MSSM Higgs LEP combination in 2001 [13], new 2-loop calculations of top loop corrections to the Higgs boson mass have become available [14]. They shift the maximal $m_{\mathrm{h}}$ achievable in the $m_{\mathrm{h}}-\max$ scenario upwards by up to $5 \mathrm{GeV}$. The maximal $m_{\mathrm{h}}$ lies at about $135 \mathrm{GeV}$. While the expected experimental lower limit on $m_{\mathrm{h}}$ for low $\tan \beta$ is not expected to change much with respect to the latest LEP exclusion (cf. Fig. 11, the theoretical upper limit on $m_{\mathrm{h}}$ shifted from the border of the black area to the border of the light grey (yellow) area. If the top mass would additionally shift upwards from its current central value of $m_{\mathrm{t}}=174.3 \pm 5.1 \mathrm{GeV}$ [15] by only one sigma (which could well be the case, given latest measurements from $\mathrm{D} 0$ in the leptonic decay channel [16]), then the upper limit on $m_{\mathrm{h}}$ would increase again by almost $5 \mathrm{GeV}$, as indicated by the border of the dark grey (red) area in Fig. 1]

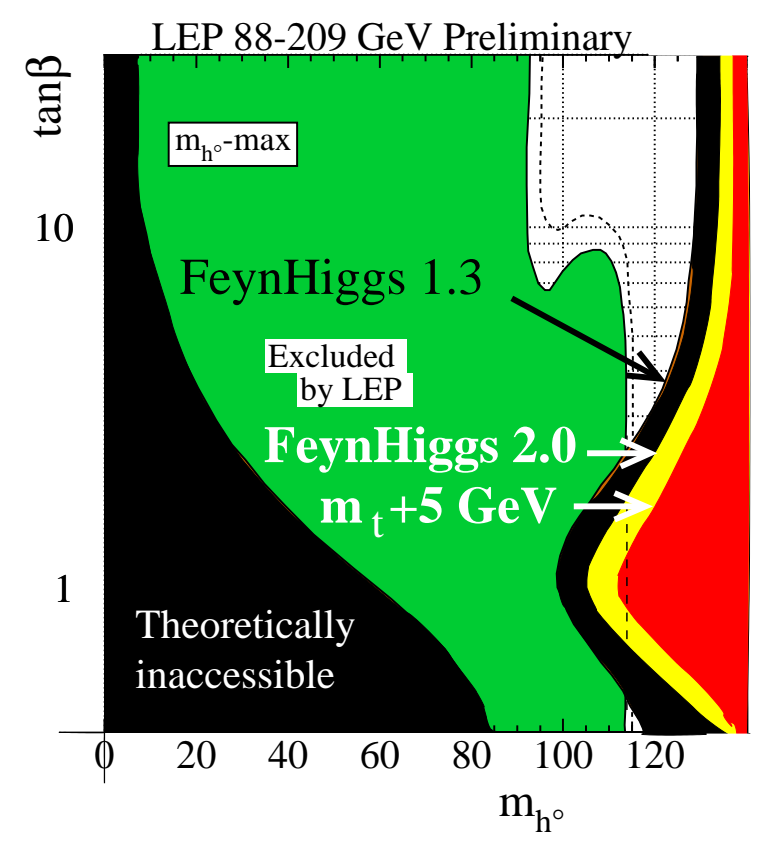

Figure 1. Expected exclusion areas of the $m_{\mathrm{h}}-\max$ scenario in the $m_{\mathrm{h}}, \tan \beta$ projection for increased upper bounds on the mass of the $\mathrm{h}$ boson. The experimental exclusion areas shown are those of the LEP combination 2001 [13]. The expected theoretical upper bounds for $m_{\mathrm{h}}$ given new order $\alpha_{\mathrm{t}}^{2}$ loop corrections to the Higgs mass is shown in light grey (yellow) and the additional effect of a possible shift in $m_{\mathrm{t}}$ by $+1 \sigma$ is overlaid in dark grey (red). The theoretically inaccessible area is forbidden by theory.

This example shows that new theoretical developments and a higher precision on $m_{\mathrm{t}}$ could well influence the exclusion of low $\tan \beta$ by LEP. A higher precision on $m_{\mathrm{t}}$ is therefore highly desireable. This could also have implication on the search channels that have to be investigated at future accelerator experiments at LHC searching for a MSSM Higgs boson, where the region of small $\tan \beta$ can not be regarded as excluded by LEP.

\section{Interpretation of the Higgs searches in the MSSM}

The combination of all Higgs searches of one experiment is used to derive [17 18 6] exclusions in the MSSM parameter space for the scenarios in Tab. 10 No major changes with respect to previous interpretations are recieved for the no-mixing, $m_{\mathrm{h}}-\max$ and large- $\mu$ scenarios. The latter now can be almost completely exluded by one experiment alone [17], thanks to flavour independent searches [19]. OPAL has also studied [6] the new CPC scenarios 4 to 7 from Tab. 1

In summary, the parameter choices of the new CPC benchmark scenarios introduce no need for new searches at LEP. Latest in a LEP combination, all topologies are covered up to the kinematic limits of the production channels. The limits on $m_{\mathrm{h}}$ and $m_{\mathrm{A}}$ are around 85 to $90 \mathrm{GeV}$ for all CPC scenarios [20 17] 186 . 


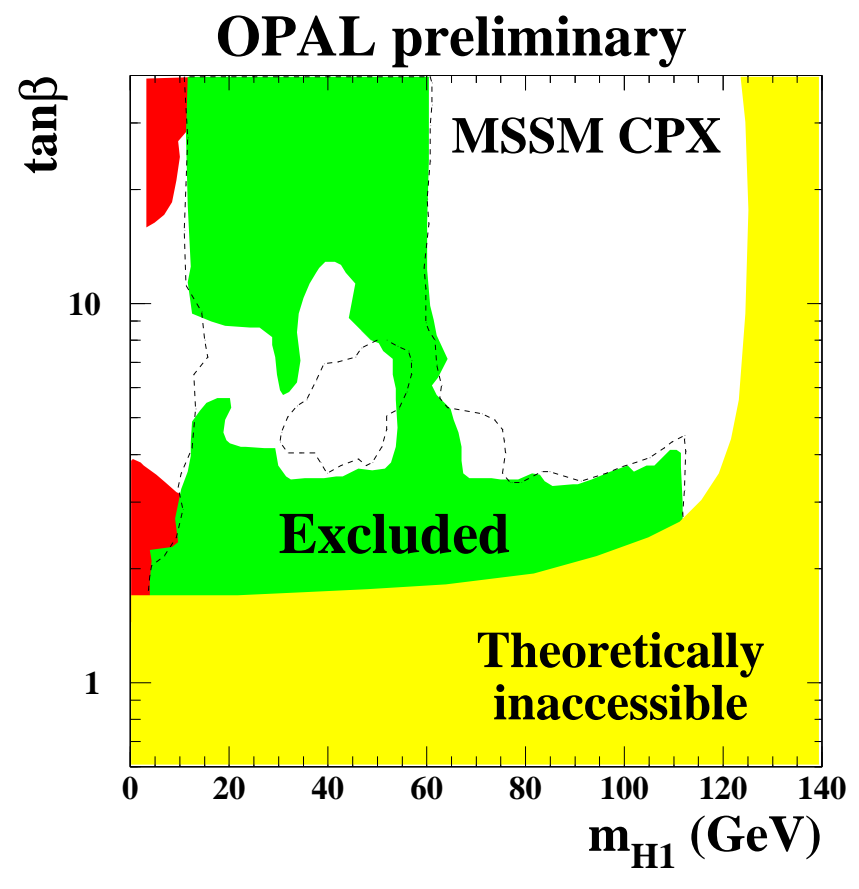

Figure 2. Exclusion area of the CPX scenario in the $\tan \beta, m_{\mathrm{H}_{1}}$ projection from OPAL. The theoretically inaccessible area is forbidden by theory.

The CPX scenario with maximal CP violation in the Higgs sector shows a decoupling of the lightest Higgs bosons $\mathrm{H}_{1}$ from the $\mathrm{Z}$ in the intermediate $\tan \beta$ range from 4 to $10\left(\mathrm{H}_{1}\right.$ and $\mathrm{H}_{2}$ being the lightest and next-to-lightest Higgs boson mass eigenstates). There anyhow $\mathrm{H}_{2}$ couples to the $\mathrm{Z}$ and is heavier than around $100 \mathrm{GeV}$. Where kinematically accessible, the decay $\mathrm{H}_{2} \rightarrow \mathrm{H}_{1} \mathrm{H}_{1}$ is dominant. Another difference to the common CPC scenarios is the large mass difference $m_{\mathrm{H}_{2}}-m_{\mathrm{H}_{1}}$ in the range with dominant pair production.

Fig. 2] [6] shows the exclusion areas of the CPX scenario. In the region with dominant $\mathrm{e}^{+} \mathrm{e}^{-} \rightarrow \mathrm{H}_{2} \mathrm{Z} \rightarrow \mathrm{H}_{1} \mathrm{H}_{1} \mathrm{Z}$ production at intermediate $\tan \beta$ and $m_{\mathrm{h}}<50 \mathrm{GeV}$ open areas emerge. It is expected that a LEP combination will be able to close these holes. Also the lower limit on $m_{\mathrm{H}_{1}}$ in the large $\tan \beta$ region, where pair production dominates, is reduced due to the large $m_{\mathrm{H}_{2}}-m_{\mathrm{H}_{1}}$. At $\tan \beta>5$ and $m_{\mathrm{H}_{1}}<10 \mathrm{GeV}$, below the $\mathrm{b} \bar{b}$ production threshold and in the pair production region, hardly any experimental constraints exist, since no pair production searches for $m_{\mathrm{H}_{2}} \approx 100 \mathrm{GeV}$ and $m_{\mathrm{H}_{1}}<10 \mathrm{GeV}$ exist. Only at large $\tan \beta>20$ Yukawa production searches can be used.

\section{Conclusions}

The developments in the MSSM Higgs searches at LEP after the end of LEP data taking in November 2000 exhibit four important lessons. First, also the increased set of CPC and CPV benchmark scenarios do not cover the full range of possible experimental phenomena in the MSSM Higgs sector. Therefore, secondly, a large variety of individual searches is necessary to cover the rich physics spectrum of the MSSM Higgs sector, which only now become fully available.

Third, new theoretical developments can influence limits on MSSM parameters. Especially the $\tan \beta$ exclusion of the final LEP combination could be affected. This is also important for the possible MSSM topologies in Higgs searches at the LHC. The importance of external measurements like $m_{\mathrm{t}}$ from the Tevatron becomes evident. A greater precision on $m_{\mathrm{t}}$ would be benefitial. Fourth, CPV scenarios show that there is still no strict lower limit on the Higgs mass from LEP. Especially in regions with low $m_{\mathrm{H}_{1}}$, but either dominant $\mathrm{e}^{+} \mathrm{e}^{-} \rightarrow \mathrm{H}_{2} \mathrm{Z}$ or dominant $\mathrm{e}^{+} \mathrm{e}^{-} \rightarrow \mathrm{H}_{1} \mathrm{H}_{2}$ production no $\tan \beta$ independent limit on the Higgs mass exists. Also these regions must probably be sought by future colliders.

\section{References}

1. A. Pilaftsis and C. E. Wagner, Nucl. Phys. B 553 (1999) 3.

2. M. Carena, S. Heinemeyer, C. E. Wagner and G. Weiglein, arXiv:hep-ph/9912223.

3. M. Carena, S. Heinemeyer, C. E. Wagner and G. Weiglein, Eur. Phys. J. C 26 (2003) 601.

4. M. Carena, J. R. Ellis, A. Pilaftsis and C. E. Wagner, Phys. Lett. B 495 (2000) 155.

5. G. Abbiendi et al. [OPAL Collaboration], Eur. Phys. J. C 27 (2003) 483.

6. OPAL Collaboration, Search for Neutral Higgs Bosons Predicted by $C P$ Conserving and $C P$ Violating MSSM Scenarios with the OPAL detector at LEP, 2003, OPAL PN524

7. [DELPHI Collaboration], Searches for invisibly decaying Higgs bosons with the DELPHI detector at LEP, DELPHI 2003036 CONF 656.

8. S. Heinemeyer, W. Hollik and G. Weiglein, Comp. Phys. Comm. 124 (2000) 76; Also see http://www. feynhiggs.de.

9. M. Frank, S. Heinemeyer, W. Hollik and G. Weiglein, hep$\mathrm{ph} / 0212037$.

Also see www . feynhiggs. de.

10. S. Heinemeyer, W. Hollik and G. Weiglein, Eur. Phys. Jour. C9 (1999) 343.

11. M. Carena, M. Quirós and C.E.M. Wagner, Nucl. Phys. B461 (1996) 407.

12. M. Carena, S. Mrenna and C. Wagner, Phys. Rev. D60 (1999) 075010

13. ALEPH, DELPHI, L3 and OPAL Collaborations, OPAL TN699

14. A. Brignole, G. Degrassi, P. Slavich and F. Zwirner, Nucl. Phys. B 631 (2002) 195;

G. Degrassi, S. Heinemeyer, W. Hollik, P. Slavich and G. Weiglein, Eur. Phys. J. C 28 (2003) 133.

15. D.E. Groom et al, Eur. Phys. J. C15 (2000) 1, available on the PDG WWW pages (URL: http: //pdg. lbl.gov/).

16. M. Coca [CDF \& D0 Collaborations], FERMILAB-CONF-03238-E Presented at Flavor Physics and CP Violation (FPCP 2003), Paris, France, 3-6 Jun 2003

17. J. Fernandez [DELPHI Collaboration], DELPHI 2003-045CONF-665, contributed paper no. 320

18. P. Achard et al. [L3 Collaboration], Phys. Lett. B 545 (2002) 30

19. M. Boonekamp, Flavour and Model Independent Higgs Searches, these proceedings

20. A. Heister et al. [ALEPH Collaboration], Phys. Lett. B 526 (2002) 191. 